Nataša A. Kablar

Vladimir Kvrgić

Dragutin Lj. Debeljković

ISSN 1333-1124

eISSN 1849-1391

\title{
SINGULARLY IMPULSIVE DYNAMICAL SYSTEMS WITH TIME DELAY: MATHEMATICAL MODEL AND STABILITY
}

UDC 517.93:512.6

\begin{abstract}
Summary
In this paper we introduce a new class of systems, the so-called singularly impulsive or generalized impulsive dynamical systems with time delay. Dynamics of these systems is characterized by a set of differential and difference equations with time delay, and by algebraic equations. They represent a class of hybrid systems where algebraic equations represent constraints that differential and difference equations with time delay need to satisfy. In this paper we present a model, assumptions about the model, and two classes of singularly impulsive dynamical systems with delay - time-dependent and state-dependent. Further, we present the Lyapunov and asymptotic stability theorems for nonlinear time-dependent and state-dependent singularly impulsive dynamical systems with time delay.
\end{abstract}

Keywords: $\quad$ singular systems, singularly impulsive dynamical systems, time delay, mathematical model, stability

\section{Introduction}

Modern complex engineering systems as well as biological and physiological systems typically possess a multi-echelon hierarchical hybrid architecture characterized by continuous-time dynamics at the lower levels of hierarchy and discrete-time dynamics at the higher levels of the hierarchy. Hence, it is not surprising that hybrid systems have been the subject of intensive research in the recent years (see Branicky et al. (1998), Ye et al. (1998 b), Haddad, Chellaboina and Kablar (2001a-b)). Such systems include dynamical switching systems, Branicky (1998), Leonessa et al. (2000), non-smooth impact and constrained mechanical systems, Back et al. (1993), Brogliato (1996), Brogliato et al. (1997), biological systems, Lakshmikantham et al. (1989), demographic systems, Liu (1994), sampled-data systems, Hagiwara and Araki (1988), discrete event systems, Passino et al. (1994), intelligent vehicle/highway systems, Lygeros et al. (1998), flight control systems, etc. The mathematical descriptions of many of these systems can be characterized by impulsive differential equations, Simeonov and Bainov (1985), Liu (1988), Lakshmikantham et al. (1989, 1994), Bainov and Simeonov (1989, 1995), Kulev and Bainov (1989), Lakshmikantham and Liu (1989), Hu et al. (1989), Samoilenko and Perestyuk (1995), Haddad, Chellaboina and Kablar (2001a-b). Impulsive dynamical systems can be viewed as a subclass of hybrid systems. 
Motivated by the results from the research on impulsive dynamical systems presented in Haddad, Chellaboina, and Kablar $(2001,2005)$, by the author's previous research on singular or generalized systems, and by the results from the research on singularly impulsive dynamical systems published in Kablar $(2003,2010)$, we presented a new class of singularly impulsive or generalized impulsive dynamical systems with time delay. The system represents a novel class of hybrid systems and a generalization of impulsive dynamical systems to incorporate the singular nature of the systems and time delays. Wide application of this class of systems can be found in contact problems and in hybrid systems.

We present a mathematical model of the singularly impulsive dynamical systems with time delay. We show how it can be viewed as a general system from which impulsive dynamical systems with time delay, singular continuous-time systems with time delay, and singular discrete-time systems with time delay, as well as without time delay, are created. Then we present assumptions needed for the model and the division of this class of systems into time-dependent and state-dependent singularly impulsive dynamical systems with time delay with respect to the resetting set. Finally, we draw some conclusions and define future work.

In this paper, we produce the Lyapunov and asymptotic stability results for the class of nonlinear singularly impulsive dynamical systems with time delay. These results are further specialized to the linear case. Note that the usual stability definitions are valid for addressing the stability of the zero solution of a singularly impulsive dynamical system. Then we draw some conclusions and define future work.

At first, we establish definitions and notations. Let $\mathbf{R}^{n}$ denote the set of real numbers, let $\mathbf{R}^{n}$ denote the set of $n \times 1$ real column vectors, let $\mathbf{N}$ denote the set of nonnegative integers, and let $I_{n}$ or $I$ denote the $n \times n$ identity matrix. Furthermore, let $\partial S, \dot{S}, \bar{S}$ denote the boundary, the interior, and a closure of the subset $S \subset \mathbf{R}^{n}$, respectively. Finally, let $C^{0}$ denote the set of continuous functions and $C^{r}$ the set of functions with $r$ continuous derivatives.

\section{Mathematical Model of Singularly Impulsive Dynamical Systems with Time Delay}

A singularly impulsive dynamical system with delay consists of three elements: 1. A possibly singular continuous-time dynamical equation with time delay, which governs the motion of the system between resetting events;

2. A possibly singular difference equation with time delay, which governs the way the states are instantaneously changed when a resetting occurs; and

3. A criterion for determining when the states of the system are to be reset.

Mathematical model of these systems is described with

$$
\begin{aligned}
& E_{c} \dot{x}(t)=f_{c}(x(t, \tau))+G_{c}(x(t, \tau)) u_{c}(t), \quad\left(t, x(t, \tau), u_{c}(t)\right) \notin S, \\
& E_{d} \Delta x(t)=f_{d}(x(t, \tau))+G_{d}(x(t, \tau)) u_{d}(t), \quad\left(t, x(t, \tau), u_{d}(t)\right) \in S, \\
& y_{c}(t)=h_{c}(t, \tau)+J_{c}(x(t, \tau)) u_{c}(t), \quad\left(t, x(t, \tau), u_{c}(t)\right) \notin S, \\
& y_{d}(t)=h_{d}(t, \tau)+J_{d}(x(t, \tau)) u_{d}(t), \quad\left(t, x(t, \tau), u_{d}(t)\right) \in S,
\end{aligned}
$$

where $t \geq 0, \tau>0, x(0)=x_{0}, x(t, \tau) \in D \subset \mathbf{R}^{n} \times \mathbf{N}, t \geq 0, D$ is an open set with $0 \in D$, inputs are $u_{c} \in U_{c} \subset \mathbf{R}^{m_{c}}, u_{d} \in U_{d} \subset \mathbf{R}^{m_{d}}, t_{k}$ denotes $k-t h$ instant of time at which $\left(t, x(t, \tau), u_{c}(t)\right)$ intersects $S$ for a particular trajectory $x(t, \tau)$ and outputs are $y_{c} \in \mathbf{R}^{l_{c}}, y_{d} \in \mathbf{R}^{l_{d}}, f_{c}: D \rightarrow \mathbf{R}^{n}$, is Lipschitz continuous and satisfies $f_{c}(0)=0, G_{c}: D \rightarrow \mathbf{R}^{n \times m_{c}}, f_{d}: D \rightarrow \mathbf{R}^{n}$ is continuous and 
satisfies $f_{d}(0)=0, G_{d}: D \rightarrow \mathbf{R}^{n \times m_{d}}, h_{c}: D \rightarrow \mathbf{R}^{l c} \quad$ and satisfies $h_{c}(0)=0, J_{c}: D \rightarrow \mathbf{R}^{l_{c} \times m_{c}}$, $h_{d}: D \rightarrow \mathbf{R}^{l_{d}}$ and satisfies $h_{d}(0)=0, J_{d}: D \rightarrow \mathbf{R}^{l_{d} \times m_{d}}$ and $S \subset[0, \infty) \times \mathbf{R}^{n} \times U_{c}$ is the resetting set. Here, as in Haddad, Chellaboina, and Kablar (2001a), we assume that $u_{c}(\cdot)$ and $u_{d}(\cdot)$ are restricted to the class of admissible inputs consisting of measurable functions $\left(u_{c}(\cdot), u_{d}(\cdot)\right) \in U_{c} \times U_{d}$ for all $t \geq 0$ and $k \in N_{[0, t)} \equiv k: 0 \leq t_{k}<t$, where the constraint set $U_{c} \times U_{d}$ is given with $(0,0) \in U_{c} \times U_{d}$. We refer to the differential equation (2.1) as the continuous-time dynamics with time delay, and we refer to the difference equation (2.2) as the resetting law.

Matrices $E_{c}, E_{d}$ may be singular matrices. In the case of $E_{c}=I, E_{d}=I$ and $\tau=0,(2.1)-$ (2.4) represent standard impulsive dynamical systems described in Haddad, Chellaboina, and Kablar (2001a), and Haddad, Kablar, and Chellaboina (2000, 2005), where stability, dissipativity, feedback interconnections, optimality, robustness, and disturbance rejection were analyzed. In the absence of discrete dynamics, they specialize to singular continuoustime systems, with further specialization of $E_{c}=I$ to standard continuous-time systems. If only discrete dynamics is present, they specialize to singular discrete-time systems, with further specialization of $E_{d}=I$ to standard discrete-time systems.

In the case of $E_{c}=I, E_{d}=I$, and $\tau \neq 0,(2.1)-(2.4)$ represent standard impulsive dynamical systems with time delay. In the absence of discrete dynamics, they specialize to singular continuous-time systems with time delay, with further specialization of $E_{c}=I$ to standard continuous-time systems with time delay. If only discrete dynamics is present, they specialize to singular discrete-time systems with time delay, with further specialization of $E_{c}=I$ to standard discrete-time systems with time delay.

Therefore, once the theory of the singularly impulsive or generalized impulsive dynamical systems with time delay is developed, it can be viewed as a generalization of the singular and the impulsive dynamical system with time delay theory, unifying them into a more general new system theory.

In the text below, a basic setting and a division of this class of systems with respect to the definition of the resetting sets are given, together with adequate assumptions needed for the model.

We make the following additional assumptions:

A1. $\left(0, x_{0}, u_{c 0}\right) \notin S$, where $x(0)=x_{0}$ and $u_{c 0}$, that is, the initial condition is not in $S$.

A2. If $\left(t, x(t, \tau), u_{c}(t)\right) \in \bar{S} \backslash S$, then there exists $\varepsilon>0$ such that, for all $0<\delta<\varepsilon$, $s(t+\delta ; t, x(t, \tau), u c(t+\delta)) \notin S$.

A3. If $\left(t_{k}, x\left(t_{k}\right), u_{c}\left(t_{k}\right)\right) \in \partial S \cap S$, then there exists $\varepsilon>0$ such that, for all $0<\delta<\varepsilon$ and $\quad u_{d}\left(t_{k}+\delta\right) \in U_{d}, s\left(t_{k}+\delta ; t_{k}, E_{d} x\left(t_{k}+\delta\right), u_{c}\left(t_{k}+\delta\right)\right) \notin S$.

A4. We assume consistent initial conditions (also prior to and after every resetting).

Assumption A1 ensures that the initial condition for resetting the differential equations (2.1) and (2.2) is not a point of discontinuity. This assumption is made for convenience. If $\left(0, x_{0}, u_{c 0}\right) \in S$, then the system initially resets to $E d x_{0}^{+}=E_{d} x_{0}+f_{d}\left(x_{0}\right)+G d\left(x_{0}\right) u_{d}$ which serves as the initial condition for the continuous dynamics (2.1). It follows from A3 that the trajectory then leaves $S$. We assume in A2 that if a trajectory reaches the closure of $\bar{S}$ at a point that does not belong to $S$, then the trajectory must be directed away from $S$, that is, a trajectory cannot enter $S$ through a point that belongs to the closure of $S$ but not to $S$. Finally, A3 ensures that when a trajectory intersects the resetting set $S$, it instantaneously exits $S$. We make the following remarks. 
Remark 2.1. It follows from A 3 that resetting removes the pair $\left(t_{k}, x_{k}, u_{c}\left(t_{k}\right)\right)$ from the resetting set $S$. Thus, immediately after resetting occurs, the continuous time dynamics (2.1), and not the resetting law (2.2), becomes the active element of the singularly impulsive dynamical system.

Remark 2.2. It follows from A1-A3 that no trajectory can intersect the interior of $\mathrm{S}$. According to A1, the trajectory $x(t)$ begins outside the set $S$. Furthermore, it follows from A2 that a trajectory can only reach $S$ through a point belonging to both $S$ and its boundary. Finally, from A3, it follows that if a trajectory reaches a point $S$ that is on the boundary of $S$, then the trajectory is instantaneously removed from $S$. Since a continuous trajectory starting outside of $S$ and intersecting the interior of $S$ must first intersect the boundary of $S$, it follows that no trajectory can reach the interior of $S$.

Remark 2.3. It follows from A1-A3 and Remark 1.2 that $\partial S \cup S$ is closed and hence the resetting times $t_{k}$ are well defined and distinct.

Remark 2.4. Since the resetting times are well defined and distinct, and since the solutions to (2.1) exist and are unique, it follows that the solutions to the singularly impulsive dynamical system (2.1), (2.2) also exist and are unique over a forward time interval.

In Haddad, Chellaboina and Kablar (2001a), the resetting set $S$ is defined in terms of a countable number of functions $n_{k}: \mathbf{R}^{n} \rightarrow(0, \infty)$ and is given by

$$
S=\bigcup_{k}\left\{n_{k}(), u_{c}\left(n_{k}(x)\right): x \in \mathbf{R}^{n}\right\}
$$

The presence of phenomena in singularly impulsive dynamical systems with time delay and with a resetting set of the form (2.5) can be quite involved. In particular, such systems exhibit Zenoness, beating, as well as confluence phenomena wherein solutions exhibit infinitely many transitions in finite times, and coincide after a given point of time, Haddad, Chellaboina and Kablar (2001a). In this paper we assume that the existence and uniqueness properties of a given singularly impulsive dynamical system with time delay are satisfied in forward time. Furthermore, since singularly impulsive dynamical systems of the form (2.1) (2.4) involve impulses at variable times, they are time-varying systems. Here, we will consider singularly impulsive dynamical systems involving two distinct forms of the resetting set $S$. In the first case, the resetting set is defined by a prescribed sequence of times which are independent of state $x$. These equations are thus called time-dependent singularly impulsive dynamical systems with time delay. In the second case, the resetting set is defined by a region in the state space that is independent of time. These equations are called state-dependent singularly impulsive dynamical systems with time delay.

\subsection{Time-Dependent Singularly Impulsive Dynamical Systems with Time Delay}

Time-dependent singularly impulsive dynamical systems with time delay can be written as (2.1)-(2.4) with $S$ defined as

where

$$
S=n \times \mathbf{R}^{n} \times U_{c}
$$

$$
n=t_{1}, t_{2}, \ldots
$$

and $0<t_{1}<t_{2}<\ldots$ are prescribed resetting times. When an infinite number of resetting times are used and $t_{k} \rightarrow \infty$ as $k \rightarrow \infty$, then $S$ is closed. Now (2.1)-(2.4) can be rewritten in the form of the time-dependent singularly impulsive dynamical system with time delay 


$$
\begin{aligned}
& E_{c} \dot{x}(t)=f_{c}(x(t, \tau))+G_{c}(x(t, \tau)) u_{c}(t), \quad t \neq t_{k}, \\
& E_{d} \Delta x(t)=f_{d}(x(t, \tau))+G_{d}(x(t, \tau)) u_{d}(t), \quad t=t_{k}, \\
& y_{c}(t)=h_{c}(x(t, \tau))+J_{c}(x(t, \tau)) u_{c}(t), \quad t \neq t_{k}, \\
& y_{d}(t)=h_{d}(x(t, \tau))+J_{d}(x(t, \tau)) u_{d}(t), \quad t=t_{k},
\end{aligned}
$$

Since $0 \in \tau$ and $t_{k}<t_{k+1}, \tau>0$, it follows that the assumptions A1-A3 are satisfied. Since time-dependent singularly impulsive dynamical systems with time delay involve impulses at a fixed sequence of times, they are time-varying systems.

Remark 2.5. The time-dependent singularly impulsive dynamical system with time delay (2.8)-(2.11), with $E_{c}=I$ and $E_{d}=I$, includes, as a special case, the impulsive control problem addressed in the literature wherein at least one of the state variables of the continuous-time plant can be changed instantaneously to any given value given by an impulsive control at a set of control instants $\tau$, Haddad, Chellaboina and Kablar (2001a).

\subsection{State-Dependent Singularly Impulsive Dynamical Systems with Time Delay}

State-dependent singularly impulsive dynamical systems with time delay can be written as (2.1)-(2.4) with $S$ defined as

$$
S=[0, \infty) \times Z,
$$

where $Z=Z_{x} \times U_{c}$ and $Z_{x} \subset \mathbf{R}^{n}$. Therefore, (2.1)-(2.4) can be rewritten in the form of the state-dependent singularly impulsive dynamical system with time delay

$$
\begin{aligned}
& E_{c} \dot{x}(t)=f_{c}(x(t, \tau))+G_{c}(x(t, \tau)) u_{c}(t), \quad(x(t, \tau)), u_{c} \notin Z, \\
& E_{d} \Delta x(t)=f_{d}(x(t, \tau))+G_{d}(x(t, \tau)) u_{d}(t), \quad\left(x(t, \tau), u_{c}(t)\right) \notin Z, \\
& y_{c}(t)=h_{c}(x(t, \tau))+J_{c}(x(t, \tau)) u_{c}(t), \quad\left(x(t, \tau), u_{c}(t)\right) \notin Z, \\
& y_{d}(t)=h_{d}(x(t, \tau))+J_{d}(x(t, \tau)) u_{d}(t), \quad\left(x(t, \tau), u_{d}(t)\right) \in Z .
\end{aligned}
$$

We assume that $\left(x_{0}, u_{c 0}\right) \notin Z, \tau>0,(0,0) \notin Z$, and that the resetting action removes the pair $\left(x, u_{c}\right)$ from the set $Z$; that is, if $\left(x, u_{c}\right) \in Z$, then $\left(E_{d} x+f_{d}(x)+G_{d}(x) u_{d}\right) \notin Z, u_{d} \in U_{d}$. In addition, we assume that if at time $t$ the trajectory $\left(x(t, \tau), u_{c}(t)\right) \in \bar{Z} \backslash Z$, then there exists $\varepsilon$ such that for $0<\delta<\varepsilon,\left(x(t+\tau+\delta), u_{c}(t+\delta)\right) \notin Z$. These assumptions represent the specialization of A1-A3 to the particular resetting set (2.12). It follows from these assumptions that for a particular initial condition, the resetting times $\tau_{k}\left(x_{0}\right)$ are distinct and well defined. Since the resetting set $Z$ is a subset of the state space and is independent of time, state-dependent singularly impulsive dynamical systems with time delay are timeinvariant systems. Finally, in the case where $S=[0, \infty) \times \mathbf{R}^{n} \times Z_{u_{c}}$, where $Z_{u_{c}} \subset U_{c}$, we refer to (2.13)-(2.16) as an input-dependent singularly impulsive dynamical system with time delay. Both these cases represent a generalization of the impulsive control problem considered in the literature. 


\section{Lyapunov and Asymptotic Stability of Singularly Impulsive Dynamical Systems with Time Delay}

In this section we present the Lyapunov and asymptotic stability results of singularly impulsive dynamical systems with time delay.

Theorem 3.1. Suppose there exists a continuously differentiable function $V: D \rightarrow[0, \infty)$ satisfying $V(0)=0, V\left(E_{c} x\right) \geq 0, x \neq 0$, and

$$
\begin{aligned}
& V^{\prime}\left(E_{c} x\right) f_{c}(x) \leq 0, \quad x \notin D, \\
& V\left(E_{d} x+f_{d}(x)\right) \leq V(x), \quad x \in D,
\end{aligned}
$$

Then the zero solution $x(t, \tau) \equiv 0$ of the undisturbed $\left(u_{c}(t), u_{d}\left(t_{k}\right)\right)=(0,0)$ time-dependent singularly impulsive dynamical system with time delay (2.8),(2.9) is Lyapunov stable. Furthermore, if the inequality (3.17) is strict for all $x \neq 0$, then the zero solution $x(t, \tau)=0$ of the undisturbed $\left(u_{c}(t), u_{d}\left(t_{k}\right)\right)=(0,0)$ time-dependent singularly impulsive dynamical system with time delay (2.8),(2.9) is asymptotically stable. If, in addition, $D=\mathbf{R}^{n}$ and

$$
V\left(E_{c} x\right) \rightarrow \infty \text { as }\|x\| \rightarrow \infty,
$$

then the zero solution $x(t, \tau)=0$ of the undisturbed $\left(u_{c}(t), u_{d}\left(t_{k}\right)\right)=(0,0)$ time-dependent singularly impulsive dynamical system with time delay (2.8), (2.9) is globally asymptotically stable, Haddad, Chellaboina, and Kablar (2001), Kablar (2003b).

Proof. Prior to the first resetting time, we can determine the value of $V(x(t, \tau))$ as

$$
\left.V\left(E_{c} x(t, \tau)\right)=V\left(E_{c} x(0)\right)+\int_{0}^{\infty} V^{\prime}\left(E_{c} x(s, \tau)\right) f_{c}(x(s, \tau))\right) d s, \quad t \in\left[0, t_{1}\right] .
$$

Between consecutive resetting times $t_{k}$ and $t_{k+1}$, we can determine the value of $V\left(E_{c} x(t, \tau)\right)$ as its initial value plus the integral of its rate of change along the trajectory $x(t, \tau)$, that is,

$$
\left.V\left(E_{c} x(t, \tau)\right)=V\left(E_{d} x\left(t_{k}\right)\right)+f_{d}\left(x\left(t_{k}\right)\right)+\int_{t}^{t_{k}} V^{\prime}\left(E_{c} x(s, \tau)\right) f_{c}(x(s, \tau))\right) d s, \quad t \in\left(t_{k}, t_{k+1}\right] .
$$

for $k=1,2, \ldots$ Adding and subtracting $V\left(E_{d} x(t, \tau)\right)$ to and from the right hand side of the (3.21) yields

$$
\begin{array}{r}
V\left(E_{c} x(t, \tau)\right)=V\left(E_{c} x\left(t_{k}\right)\right)+\left[V\left(E_{d} x\left(t_{k}\right)\right)+f_{d}\left(x\left(t_{k}\right)\right)-V\left(E_{d} x\left(t_{k}\right)\right)\right] \\
\left.+\int_{t}^{t_{k}} V^{\prime}\left(E_{c} x(s, \tau)\right) f_{c}(x(s, \tau))\right) d s, \quad t \in\left(t_{k}, t_{k+1}\right]
\end{array}
$$

and in particular at time $t_{k+1}$,

$$
\begin{aligned}
V\left(E_{d} x\left(t_{k+1}\right)\right)=V( & \left.E_{d} x\left(t_{k}\right)\right)+\left[V\left(E_{d} x\left(t_{k}\right)\right)+f_{d}\left(x\left(t_{k}\right)\right)-V\left(E_{d} x\left(t_{k}\right)\right)\right] \\
& \left.+\int_{t}^{t_{k+1}} V^{\prime}\left(E_{c} x(s, \tau)\right) f_{c}(x(s, \tau))\right) d s, \quad t \in\left(t_{k}, t_{k+1}\right] .
\end{aligned}
$$

By recursively substituting (3.23) into (3.22) and ultimately into (3.20), we obtain

$$
\begin{aligned}
\left.V\left(E_{c} x(t, \tau)\right)=V\left(E_{c} x(0)\right)+\int_{0}^{t} V^{\prime}\left(E_{c} x(s, \tau)\right) f_{c}(x(s, \tau))\right) d s \\
+\sum_{i=1}^{k}\left[V\left(E_{d} x\left(t_{i}\right)+f_{d}\left(x\left(t_{i}\right)\right)\right)-V\left(E_{d} x\left(t_{i}\right)\right)\right] .
\end{aligned}
$$

If we allow $t_{0}=0$, and $\sum_{0}^{i=1}=0$, then (3.24) is valid for $k \in N$. 
From (3.24) and (3.18) we obtain

$$
\left.V\left(E_{c} x(t, \tau)\right)=V\left(E_{c} x(0)\right)+\int_{0}^{t} V^{\prime}\left(E_{c} x(s, \tau)\right) f_{c}(x(s, \tau))\right) d s, \quad t \geq 0 .
$$

Furthermore, it follows from (3.17) that

$$
V\left(E_{c} x(t, \tau)\right) \leq V\left(E_{c} x(0)\right), \quad t \geq 0
$$

so that the Lyapunov stability follows from standard arguments. Next, it follows from (3.18) and (3.24) that

$$
\left.V\left(E_{c} x(t, \tau)\right)-V\left(E_{c} x(s, \tau)\right) \leq \int_{s}^{t} V^{\prime}\left(E_{c} x(s, \tau)\right) f_{c}(x(s, \tau))\right) d s, \quad t \geq s,
$$

and, assuming strict inequality in (3.17), we obtain

$$
V\left(E_{c} x(t, \tau)\right)<V\left(E_{c} x(s, \tau)\right), \quad t>s,
$$

provided $x(s, \tau) \neq 0$. Asymptotic stability, and, with (3.19), global asymptotic stability, then follow from standard arguments.

Remark 3.1. If in Theorem 3.1 the inequality (3.18) is strict for all $x(t) \neq 0$ as opposed to the inequality (3.17), and an infinite number of resetting times are used, that is, the set $\tau=\left\{t_{1}, t_{2}, \ldots\right\}$ is infinitely countable, then the zero solution $x(t, \tau) \equiv 0$ of the undisturbed timedependent singularly impulsive dynamical system with time delay (2.8), (2.9) is also asymptotically stable. A similar remark holds for Theorem 2.2.

Remark 3.2. In the proof of Theorem 3.1, we note that assuming strict inequality in (3.17), the inequality (3.28) is obtained provided $x(s, \tau) \neq 0$. This proviso is necessary since it may be possible to reset the states to the origin, in which case $x(s, \tau)=0$ for a finite value of $s$. In this case, for $t>s$, we have $V\left(E_{c} x(t, \tau)\right)=V\left(E_{c} x(s, \tau)\right)=V(0)=0$. This situation does not present a problem, however, since reaching the origin in finite time is a stronger condition than reaching the origin as $t \rightarrow \infty$.

Remark 3.3. If, additionally, in Theorem 3.1 there exist scalars $\alpha, \beta, \varepsilon>0$, and $p \geq 1$, such that $\alpha\|x\|_{p} \leq V\left(E_{c} x\right) \leq \beta\|x\|_{p}, \quad x \in D, \quad$ and (3.17) is replaced by $V^{\prime}\left(E_{c} x\right) f_{c}(x) \leq-\varepsilon V\left(E_{c} x\right), \quad x \in D$, then the zero solution $x(t, \tau)=0$ of the undisturbed $\left(u_{c}(t), u_{d}\left(t_{k}\right)\right)=(0,0)$ time-dependent singularly impulsive dynamical system with time delay (2.8), (2.9) is exponentially stable. A similar remark holds for Theorem 2.2.

Remark 3.4. Theorem 3.1 presents sufficient conditions for time-dependent singularly impulsive dynamical systems with time delay in terms of Lyapunov functions that do not depend explicitly on time. Since time-dependent singularly impulsive dynamical systems are time-varying, Lyapunov functions that explicitly depend on time can also be considered. However, in this case, the conditions on the Lyapunov functions required to guarantee stability are significantly harder to verify. For further details see Bainov and Simeonov (1989), Samoilenko and Perestyuk (1995), Ye, Michael, and Hou (1998).

Next, we state a stability theorem for nonlinear state-dependent singularly impulsive dynamical systems with time delay. 
Theorem 3.2. Suppose there exists a continuously differentiable function $V: D \rightarrow[0, \infty)$ satisfying $V(0)=0, V\left(E_{c} x\right) \geq 0, x \neq 0$, and

$$
\begin{aligned}
& V^{\prime}\left(E_{c} x\right) f_{c}(x) \leq 0, \quad x \notin Z_{x} \\
& V^{\prime}\left(E_{d} x+f_{d}(x)\right) \leq V\left(E_{c} x\right), \quad x \in Z_{x} .
\end{aligned}
$$

Then the zero solution $x(t, \tau) \equiv 0$ of the undisturbed $\left(u_{c}(t), u_{d}\left(t_{k}\right)\right)=(0,0)$ state-dependent singularly impulsive dynamical system with time delay (2.13), (2.14) is Lyapunov stable. Furthermore, if the inequality (3.29) is strict for all $x \neq 0$, then the zero solution $x(t, \tau) \equiv 0$ of the undisturbed $\left(u_{c}(t), u_{d}\left(t_{k}\right)\right)=(0,0)$ state-dependent singularly impulsive dynamical system with time delay (2.13), (2.14) is asymptotically stable. If, in addition, $D=\mathbf{R}^{n}$ and (3.19)is satisfied, then the zero solution $x(t, \tau) \equiv 0$ of the undisturbed $\left(u_{c}(t), u_{d}\left(t_{k}\right)\right)=(0,0)$ statedependent singularly impulsive dynamical system with time delay (2.13), (2.14) is globally asymptotically stable, Haddad, Chellaboina, and Kablar (2001), Kablar (2003b).

Proof. For $S=[0, \infty) \times Z_{x}$ it follows from Assumptions A1-A3 that the resetting times $n_{k}\left(x_{0}\right)$ are well defined and distinct for every trajectory of (2.13), (2.14) with $\left(u_{c}(t), u_{d}\left(t_{k}\right)\right)=(0,0)$. Now, the proof follows as in the proof of Theorem 3.1 with $t_{k}$ replaced by $n_{k}\left(x\left(t_{k}\right)\right)$.

Remark 3.5. To examine the stability of linear state-dependent singularly impulsive dynamical systems with time delay, set $f_{c}(x)=A_{c} x$, and $f_{d}(x)=\left(A_{d}-E_{d}\right) x$ in Theorem 3.2. Considering the quadratic Lyapunov function candidate $V\left(E_{c / d} x\right)=x^{T} E_{c / d}^{T} P E_{c} x$, for the argument $E_{c} x$ and $E_{d} x$, respectively, where $P>0$, from Theorem 3.2 it follows that the conditions

$$
\begin{aligned}
& x^{T}\left(A_{c}^{T} P E_{c}+E_{c}^{T} P A_{c}\right) x<0, \quad x \notin Z_{x}, \\
& x^{T}\left(A_{d}^{T} P A_{d}+E_{d}^{T} P E_{d}\right) x \leq 0, \quad x \in Z_{x},
\end{aligned}
$$

establish asymptotic stability for linear state-dependent singularly impulsive dynamical systems with time delay. These conditions are implied by $P>0, A_{c}^{T} P E_{c}+E_{c}^{T} P A_{c}<0$, and $A_{d}^{T} P A_{d}+E_{d}^{T} P E_{d} \leq 0$, which can be solved using the Linear Matrix Inequality (LMI) feasibility problem, Boyd et al. (1994). See also Haddad, Chellaboina, and Kablar (2001a).

\section{Conclusion}

Modern complex engineering systems as well as biological and physiological systems typically possess a multi-echelon hierarchical hybrid architecture characterized by continuous-time dynamics at the higher levels of the hierarchy and discrete-time dynamics at the lower levels of the hierarchy, which can be represented as hybrid and impulsive dynamical systems. In this paper we introduced a new class of systems, the so-called singularly impulsive or generalized impulsive dynamical systems with time delay. Dynamics of these systems is characterized by the set of differential and difference equations with time delay, and by algebraic equations. They represent a class of hybrid systems where algebraic equations represent constraints that differential and difference equations with time delay need to satisfy. In this paper we presented a model, assumptions about the model, and two classes of singularly impulsive dynamical systems with delay - time-dependent and state-dependent. Further, we present the Lyapunov and asymptotic stability theorems for nonlinear timedependent and state-dependent singularly impulsive dynamical systems with time delay. 


\section{Future Work}

It is left to develop an invariant-set theorem for singularly impulsive dynamical systems. Next, further work will concentrate on specializing these results and on developing time-delay systems. The latter is motivated by the recognized need in biological applications. On the other hand, finite-time and practical stability results will be produced for the class of impulsive and singularly impulsive dynamical systems with delay.

\section{Acknowledgment}

The author is grateful to all that were supportive and creative in achieving these results.

\section{REFERENCES}

[1] Bainov D.D. and P.S. Simeonov, Systems with Impulse Effect: Stability Theory and Applications. England, Ellis Horwood Limited, 1989.

[2] Boyd S., L.E. Ghaoui, E. Feron, and V. Balakrishnan, Linear Matrix Inequalities in System and Control Theory. In: SIAM studies in applied mathematics, 1994.

[3] Back A., J. Guckenheimer, and M. Myers, "A dynamical simulation facility for hybrid systems," In R. Grossman, A. Nerode, A. Ravn and H. Rischel (Eds), Hybrid Systems, New York: Springer-Verlag, pp. 255-267, 1993.

[4] Branicky M. S., "Multiple-Lyapunov functions and other analysis tools for switched and hybrid systems," IEEE Transactions on Automatic Control, Vol. 43, pp. 475-482, 1998.

[5] Branicky M. S., V. S. Borkar, and S. K. Mitter, ”A unified framework for hybrid control: model and optimal control theory," IEEE Transactions on Automatic Control, Vol. 43, pp. 31-45, 1998.

[6] Brogliato B., Non-smooth Impact Mechanics: Models, Dynamics and Control, London: Springer-Verlag, 1996.

[7] Brogliato B., S. I. Niculescu, and P. Orhant, "On the control of finitedimensional mechanical systems with unilateral constraints," IEEE Transactions on Automatic Control, Vol. 42, pp. 200-215, 1997.

[8] Haddad W.M., V.Chellaboina, N.A. Kablar, "Nonlinear Impulsive Dynamical Systems: Stability and Dissipativity," Proc. IEEE Conf. Dec. Contr., pp. 5158-5163, Phoenix, AZ, 1999a. Also in: Int. J. Contr., vol. 74, pp. 1631-1658, 2001 a.

[9] Haddad W.M., V.Chellaboina, N.A. Kablar, ”Nonlinear Impulsive Dynamical Systems: Feedback Interconnections and Optimality,” Proc. IEEE Conf. Dec. Contr., Phoenix, AZ, 1999b. Also in: Int. J. Contr., vol. 74, pp. 1659-1677, 2001 b.

[10] Haddad W.M., N.A. Kablar, V.Chellaboina, "Robustness of Uncertain Nonlinear Impulsive Dynamical Systems," Proc. IEEE Conf. Dec. Contr., Sidney, pp. 2959-2964, Australia, 2000. Also in: Nonlinear Anal., submitted.

[11] Haddad W.M., N.A. Kablar, V.Chellaboina, ”Optimal Disturbance Rejection of Nonlinear Impulsive Dynamical Systems," Nonlinear Anal., published, 2005.

[12] Kablar N.A., "Singularly Impulsive or Generalized Impulsive Dynamical Systems," Proc. Amer. Contr. Conf., Denver, CO, 2003a.

[13] Kablar N.A., "Lyapunov and Asymptotic Stability of Singularly Impulsive Dynamical Systems," Proc. IEEE Conf. Dec. Contr., USA, 2003 b.

[14] Kablar N.A., "Finite-Time Stability of Singularly Impulsive Dynamical Systems," IEEE Conf. Decision and Control, Atlanta, USA, 2010.

[15] Kablar N.A., "Robust Stability Analyse of Singularly Impulsive Dynamical Systems," Proc. Amer. Contr. Conf., USA, 2006.

[16] Lakshmikantham V., D. D. Bainov, and P. S. Simeonov, Theory of Impulsive Differential Equations, Singapore: World Scientic, 1989.

[17] Lakshmikantham V. and X. Liu, "On quasi stability for impulsive differential systems," Non. Anal. Theory, Methods and Applications, Vol. 13, pp. 819-828, 1989.

[18] Lakshmikantham V., S. Leela, and S. Kaul, "Comparison principle for impulsive differential equations with variable times and stability theory," Non. Anal. Theory, Methods and Applications, Vol. 22, pp. 499-503, 1994. 
[19] Leonessa A., W. M. Haddad, and V. Chellaboina, Hierarchical Nonlinear Switching Control Design with Applications to Propulsion Systems, London: Springer- Verlag, 2000.

[20] Liu X., "Stability results for impulsive differential systems with applications to population growth models," Dynamic Stability Systems, Vol. 9, pp. 163-174, 1994.

[21] Lygeros J., D. N. Godbole, and S. Sastry, "Verified hybrid controllers for automated vehicles," IEEE Transactions on Automatic Control, Vol. 43, pp. 522-539, 1998.

[22] Passino K.M., A. N. Michel, and P. J. Antsaklis, "Lyapunov stability of a class of discrete event systems," IEEE Transactions on Automatic Control, Vol. 39, pp. 269-279, 1994.

[23] Raibert M. H, Legged Robots that Balance, MIT Press, Cambridge, MA, 1986. [25] Samoilenko A. M. and N.A. Perestyuk, Impulsive Differential Equations. World Scientific, 1995.

[24] Ye H., A.N. Michel, and L. Hou, "Stability Analysis of Systems with Impulsive Effects," IEEE Trans. Autom. Contr., vol. 43, pp. 1719-1723, 1998.

Submitted: $\quad 26.8 .2012$

Accepted: $\quad 10.10 .2013$
Prof. Dr. Nataša A. Kablar

Lola Institute

Kneza Viseslava 70a

11000 Belgrade, Serbia

and Faculty of Computer Science

Knez Mihailova 6,

11000 Belgrade, Serbia

Dr. Vladimir Kvrgić

Lola Institute

Kneza Viseslava 70a

11000 Belgrade, Serbia

Prof. Dr. Dragutin Lj. Debeljković

Faculty of Mechanical Engineering

Kraljice Marije 16

11000 Belgrade, Serbia 